\title{
LA DONNA LEOPARDO
}

\author{
di Gianfranco Bettin Lattes
}

Gianfranco Bettin Lattes

Universidad de Florencia

Il racconto che qui viene pubblicato è stato ispirato, involontariamente, da Fermín ed è giusto ricordare brevemente in quali circostanze ho avuto da lui questo regalo. Nell'inverno del 2004, non ubbidendo ad una saggia esortazione di Seneca: Animus debes mutare non caelum! ero fuggito da Firenze a Madrid. Lì fui accolto da due amici Fermín Bouza Ảlvarez ed Antonio Alaminos Chica. Una sera ci ritrovammo insieme seduti al tavolo di una birreria. Fermín, che ben sapeva del profondo dolore che mi trascinavo dietro, per distrarmi, tra un boccale di birra e l'altro, raccontò una strana storia che gli era accaduta qualche mese prima. Aveva ricevuto una telefonata da una lettrice sconosciuta che gli dichiarava tutta la sua ammirazione per un libro a sfondo psico-sociologico sulle emozioni recentemente pubblicato da Firmín e gli chiedeva con eccessiva insistenza di incontrarlo. A questo punto io ed Antonio, punti dall'invidia, ci aspettavamo l'inizio di una classica avventura amorosa extraconiugale. Invece Fermín con la finezza tipica del poeta e del novelliere di razza disegnò il racconto sul filo delicato dei sentimenti più intimi. Era andato all'appuntamento in un hotel lussuoso insieme alla moglie Carmen Pena López che si era fermata ad aspettarlo fuori, in auto. La lettrice misteriosa, un' avvenente e ricca imprenditrice portoghese, si vide respingere con garbo le profferte affettuose ed audaci. Il risultato dell'incontro fu che Fermín, pur lusingato del felice impatto del suo libro, rivisse con estrema chiarezza tutta la forza e la bellezza del legame che aveva con Carmen, la compagna della sua vita, ed uscì dall'hotel più allegro che mai. Uno stato d'animo che rispondeva alla sua natura più autentica, come ebbe a dire di lui proprio Carmen, ricordandolo nelle pagine di GaliciaDigital: «le acompañó siempre una alegría interior profunda y un sentido del humor invencible». Qualche mese dopo esser ritornato a Firenze avevo bisogno di rivivere in qualche modo questa storia e di ritrovare quella dimensione di magia 
imprevista e sottile che Fermín aveva ricreato quella sera a beneficio dei suoi due amici, Antonio e Gianfranco, poveri professori senza ammiratrici. Così è nata La donna leopardo che naturalmente ricontestualizza i personaggi e li adatta al mio profilo, ben più modesto. Mi piace ricordare che Fermín qualche anno dopo, letta la versione fiorentina dei fatti, si era assai divertito.

«In breve possiamo dire che la lezione di Max Weber sulle origini del capitalismo è un dato illuminante per comprendere i percorsi che intraprende oggi la globalizzazione». Con queste parole, apparentemente chiare ma non prive di possibili,ulteriori problematizzazioni, il professor Sebastiano Portinari portava a termine - finalmente- il suo corso di sociologia teorica e poteva andarsene in vacanza.

Diversamente da quello che accadeva qualche anno prima, la fine delle lezioni non era stata salutata dall'applauso del gruppo di studenti che lo seguiva per l'intero anno. «I tempi sono cambiati -pensò Sebastiano, mentre lasciava l'aula con dentro l'anima un grumo di sentimenti dove sollievo e frustrazione si intrecciavano con esiti non del tutto positivi - «oppure sono cambiato io?». Da qualche mese aveva la netta sensazione di stare in quelle stanze della facoltà fiorentina di sociologia così, come se stesse in un sogno obbligato. Parlava,parlava,parlava seguendo meccanicamente il brogliaccio di un copione scritto e riscritto ma non più aggiornato. Il punto era che si stava ripiegando su sé stesso e che la cosiddetta vocazione all'educazione delle nuove generazioni si era volatilizzata. Era arrivato anche lui a quella boa, difficile da girare, che gli insegnanti di mezza età conoscono molto bene in cui si fa un bilancio e si vede come tutto, o quasi tutto, sia stato vacuo: quello che rimane è solo la conquista di un magro stipendio, uno stipendio da sopravvissuti.

Sebastiano Portinari però il suo asso nella manica se lo era tenuto con cura e nella massima riservatezza: solo sua moglie sapeva di questo segreto. Negli ultimi cinque anni aveva passato quasi tutte le sere, fatta eccezione per il sabato dedicato alla consorte (cena fuori casa, cinema e poi un po' di sesso routiniero), alla stesura di un lungo saggio sull'amore nel mondo moderno che, proprio per quella primavera sarebbe stato pubblicato da una famosa casa editrice. Oggi gli era arrivata in visione una bozza della copertina, molto elegante, che proponeva una foto di Marilyn Monroe giovane, in un atteggiamento decisamente seducente.

Erano le cinque del pomeriggio di un giorno di autunno. Le foglie del parco di platani giganti che circondavano la casa erano inesorabilmente ingiallite in attesa di tempi migliori, mentre seduto sulla poltrona del suo studio il professor 
Portinari sorseggiava il solito tè insieme a tre o quattro biscottini che venivano inzuppati con eleganza meticolosa nella tazza di porcellana cinese. A quell'ora dava un'occhiata alla posta cartacea perché preferiva sbrigare dopo la cena la posta elettronica, fatta di scambi di notizie e di pettegolezzi accademici con i colleghi italiani e stranieri. Negli ultimi due mesi, con la pubblicazione del libro sull'amore, la posta si era infittita. Era il suo editore a spedirgli ogni giovedì un pacchetto che conteneva, di solito, tra le dieci e le quindici lettere. Sebastiano Portinari era stato molto contento della risposta che il pubblico non universitario aveva dato al suo libro. Era una specie di prova del nove che si poteva trattare con serietà e con un linguaggio impegnativo un tema che riguardava tutti, giovani e vecchi, uomini e donne, ricchi e poveri e a tutte le latitudini. Nelle lettere dei lettori ti imbattevi in storie strane, a volte drammatiche, a volte assurde. Tutte convergevano nella constatazione che l'amore è un'esperienza rara, poco duratura ma insostituibile. Le storie venivano raccontate soprattutto da lettori donne, come se gli uomini fossero impediti da un ritegno virile a parlare di amore. Più probabilmente non erano degli accaniti lettori di saggi sull'amore. Insomma su dieci lettere solo una proveniva da un maschio il quale risultava sempre abbandonato dalla fidanzata o dalla moglie. Nel pacchetto di lettere di quella settimana una spiccava per l'eleganza della busta e per uno strano profumo che emanava. Sebastiano, incuriosito, lesse prima di tutto sul retro della busta il nome e l' indirizzo del mittente scritti a mano, in un corsivo assai elegante: Liliana Terzi, via Montenapoleone 8, Milano. A questo punto, con il tagliacarte d'argento che sua madre gli aveva regalato quando aveva passato l'esame di maturità classica con il massimo dei voti e con una menzione speciale, si apprestò ad aprire la missiva infilando prima la punta della lama nell'angolo sinistro della busta. Poi proseguì sbranando, con lenta delicatezza, i lembi a triangolo che la chiudevano,come se si trattasse di aprire uno scrigno foderato di seta.

“Caro Professore vorrà perdonarmi per queste poche parole. Ho letto il suo libro: mi ha aperto un nuovo mondo! La prego, mi conceda un appuntamento per una conversazione. Vengo dove e quando Lei vuole. La saluto con ossequio ed infinita ammirazione" L.T.

Seguiva un post-scriptum lapidario, con il numero del telefono cellulare.

La prima reazione di Sebastiano fu di lusingato compiacimento. Poi la ridda di ipotesi su quella lettrice, alimentata dalla curiosità e da un inveterato narcisismo, si impadronì dei suoi pensieri nelle ore successive. A cena, quella sera, si sorprese a pensare se raccontare o meno della lettera a Teresa, sua moglie, come se ci fosse da nasconderle una scappatella. Il racconto lo fece, ma solo dopo aver ingerito l'insalata mista con tonno che era al centro del loro pasto frugale per evitare l'eccesso di grassi promosso da una vita troppo sedentaria. Teresa, che conosceva Sebastiano anche in quelle pieghe della sua anima che lui non aveva 
mai esplorato, subito dopo aver sentito della lettera, lo guardò con calma serena negli occhi e poi, gli diede un ordine affettuoso: «Chiamala!». Sebastiano era uno studioso abbastanza serio ma dell'amore sapeva assai poco perché, oltre a Teresa, le sue esperienze empiriche si confinavano a due o tre compagne di università e a qualche fuggevole rapporto prezzolato con delle signorine di strada. Di quell'imperativo blandamente categorico della moglie - le cui profonde motivazioni, nella sua banalità di maschio, ignorò sempre - fu contento perché gli toglieva una vago senso di colpa che lo turbava.

Un martedì mattina, dal suo ufficio all'università, fece la telefonata. Erano da poco passate le 10. Dopo qualche squillo dall'altra parte rispose una voce calda:« Chi mi chiama?». Sebastiano si presentò educatamente e ricordando tutti i dati utili alla circostanza. Ci fu una breve pausa di silenzio, poi la voce diventò non solo calda ma avvolgente in un modo quasi imbarazzante. In breve si decise che nel fine settimana lei sarebbe venuta a Firenze. L'appuntamento era per il sabato pomeriggio nello spiazzo davanti alla basilica di San Miniato al Monte. Era stato lui a scegliere un luogo all'aperto, era stata lei a fissare il giorno e l'ora: le 16,30. Sebastiano raccontò tutto a Teresa e notò come lei fosse completamente indifferente: rispondeva con diradati monosillabi alla notizia che, in fondo, non era poi così scontata. Per quello stesso sabato lui e Teresa avevano fatto un progetto di andare a Viareggio per fare una passeggiata sulla spiaggia: una cosa che la moglie amava molto e che rappresentava uno dei rari momenti di svago che lui le concedeva. Ma Teresa non avanzò la minima protesta per questa defezione assai poco gentile.

L.T. aveva preannunciato il suo arrivo da Milano in auto e così Sebastiano pensò di aspettarla allo slargo antistante all'ingresso delle Porte Sante dove lei avrebbe potuto posteggiare comodamente. Poi, insieme in pochi passi, sarebbero saliti allo spiazzo da cui si poteva ammirare il panorama sull'intera città. L'estate era ormai finita e l'autunno non era ancora cominciato. I pomeriggi erano freschi e luminosi ed anche quel sabato Firenze si distendeva, con una struggente dolcezza, ai piedi di chi la guardava da San Miniato. Sebastiano si era vestito in un modo sobrio: giacca e cravatta come se andasse a fare una lezione, però si era spruzzato addosso un profumo di Armani, cosa che avveniva raramente, per non dire mai. Più che curioso si sentiva emozionato; si era messo in attesa verso le 16,15. Ed ecco che allo scoccare in punto della mezz'ora una Mercedes 220 nera svoltò dalla curva che approdava al parcheggio. C'era un autista con tanto di divisa che,con felinità professionale, impedì a Sebastiano di fare quel che avrebbe voluto: aprire con gentilezza la portiera da cui il passeggero doveva scendere. 
Liliana Terzi era una donna bella, bella al di sopra di ogni aspettativa. Ancora giovane, non aveva sicuramente più di 45 anni. Alta e slanciata con un sorriso smagliante offrì a Sebastiano la mano da baciare. Congedato l'autista,si avviarono come era naturale che fosse verso il grande arco in pietra e poi verso la breve salita che li avrebbe portati nel piazzale antistante alla chiesa. Sebastiano ora era confuso e quasi spaventato dall'incontro. Fu lei a condurre il gioco con signorile disinvoltura. Seduti sul muretto panoramico Liliana raccontò della sua vita di divorziata, dei tradimenti del marito,della disperazione figlia della solitudine in cui si era improvvisamente trovata e, poi, di come la lettura del libro di Sebastiano le avesse fatto capire che l'amore è una conquista quotidiana fatta di passione ma anche di rispetto, di delicatezza, di rinunce e soprattutto di un impegno continuo verso l'altro. Insomma le ore passate su quelle pagine l'avevano consolata, di più le avevano restituito una speranza di cui lei gli era profondamente grata. Poi gli raccontò che era ricchissima, che passava molti mesi dell'anno all'estero e che sentiva il bisogno di avere una specie di consigliere, qualcuno esperto e fidato a cui appoggiarsi per un sostegno psicologico, qualcuno che, come dire, pilotasse le sue emozioni. Secondo lei, lui era la persona giusta ed ora che l'aveva visto di persona sentiva, senza ombra di dubbio, che proprio lui avrebbe potuto svolgere benissimo un ruolo così delicato. Ovviamente il tempo speso per farle da tutor emotivo sarebbe stato compensato: che fissasse lui la cifra.

Sebastiano era interdetto: la bellezza di lei lo aveva colpito; si scoprì a guardarle le gambe ed il seno. Erano perfetti. Cercò di guadagnare tempo; pensava che un lavoro di questo tipo andava conciliato con il suo impegno di insegnante e di studioso, cosa non così semplice e glielo disse. Lei, sempre sorridendo, gli rispose che non vedeva dove fosse il problema. Tutto quello che chiedeva era di potergli telefonare quando voleva e poi di incontrarlo per un colloquio, con calma vis -à-vis, per lo meno un giorno al mese. Fatta la proposta sempre con un savoir-faire affabile -ma vagamente manageriale- lei si congedò, dicendo che doveva vedere alcuni amici fiorentini e che avrebbe aspettato una sua decisione per i prossimi giorni.

Nei giorni successivi Sebastiano si sentì immerso in una strana atmosfera. Gli sembrava di essere tornato giovane, aveva addosso una strana voglia di indipendenza, non rispettava più gli orari canonici che scandivano, in una maniera pressoché prussiana, la sua vita di docente universitario. Aveva ricercato L.T ed aveva accettato la sua proposta. Per eleganza non aveva voluto dirle al telefono la cifra che pretendeva, anche se aveva pensato a qualcosa che assomigliava alla metà del suo stipendio mensile ma, soprattutto aveva una voglia quasi ossessiva di rivederla. Naturalmente aveva informato Teresa del primo incontro a San Miniato e poi della decisone di farsi carico professionalmente di quella 
strana alunna, ma non l'aveva affatto consultata su questi punti e soprattutto non si curava del persistente silenzio della moglie su tutta la vicenda. Si era preoccupato, invece, di rinnovare il suo guardaroba che era non solo poco giovanile ma di mediocre qualità. Fu così che una parte non piccola dei suoi risparmi si trasformò in un completo grigio misto lana griffato, in cinque splendide camicie ed in un paio di mocassini Tod's marrone chiaro che aveva desiderato per anni.

Il secondo appuntamento fu di nuovo a Firenze. Questa volta nel suo studio all'università. L'aveva portata là, pur sapendo bene che il luogo era piuttosto squallido, perché aveva voglia che qualcuno dei suoi colleghi li incontrasse insieme,quasi che la volesse esibire come prova che lui era un professore di successo. Lei arrivò come la prima volta a bordo del suo macchinone nero, targato Milano e autista-munito. Si muoveva con una disinvoltura incredibile quasi fosse a casa sua, prima nei corridoi del dipartimento e poi al bar affollato da studenti e da docenti che la guardavano come se fosse un animale raro. Lui era con ai piedi i mocassini Tod's, indossava una camicia azzurra a righe bianche: giacca e cravatta erano scomparsi con una evidente sorpresa anche della barista che era abituato a vederlo come un docente giovanile ma grigio e serioso, anche nel modo di vestire. La conversazione si sviluppò su un piano di carattere tecnico. Lei gli raccontò che era corteggiata da un facoltoso industriale, amico dell' ex marito ma che non sentiva nessuna reale attrazione per lui. Però stava per cedergli per fuggire alla noia melanconica di una vita da single. Mentre gli parlava, lo chiamava sempre professore e gli dava del lei, ma lo occhieggiava in un modo un po' sbarazzino e quando ormai erano rimasti soli nell'ufficio di lui arrivò alla confidenza di prendergli una mano e di accarezzargliela. Come risposta al contatto improvviso ed imprevisto Sebastiano arrossì; non gli succedeva da molti anni e sentì dentro una emozione forte, quasi la stessa emozione che gli aveva provocato il primo bacio dato a Teresa. Non poté fare a meno di comparare i due eventi ed il paragone, non voluto, gli procurò una acuta sensazione di disagio che non lo lasciò più per tutto il resto dell'incontro. Nonostante tutto fece il suo lavoro di supporter emotivo con scrupolo e con impegno. La sua ricetta era che l'amore non è negoziabile,che è un fatto spontaneo, che va atteso e che non va mai abbandonata la speranza di incontrarlo. Pericoloso per il proprio equilibrio emotivo sarebbe stato, invece, prendere degli impegni in maniera non sincera e forzata per fronteggiare un problema quello della solitudine che andava valutato positivamente come la fase di attesa di un ciclo di vita successivo,che poteva essere pieno di soddisfazioni sentimentali, genuine ed appaganti. Dunque il suo consiglio era di resistere e di apprezzare il corteggiamento in una chiave pragmatica e strumentale, solo perché ciò la confermava nel suo evidente valore di donna bella, intelligente ed elegante, piena di sensibilità e di charme. Non si accorgeva, però, nel dirle queste cose, che lei era visibilmente compiaciuta degli aggettivi che lui adoperava e che lo guardava sem- 
pre più insistentemente con uno sguardo dove l'allusione ad una complicità tra di loro diventava al limite del compromettente. Lasciandosi lei gli disse che il terzo incontro, al più tardi, sarebbe stato fra tre settimane e non più a Firenze e che lo avrebbe chiamato a breve per i dettagli. Ciò detto, come se fosse un gesto abituale, lasciò una busta bianca sulla scrivania e, sempre sorridendo, dopo averlo baciato su una guancia, se ne andò. Sebastiano restò più turbato del bacio che dell'entità dell'assegno che, peraltro,era al di là di ogni ragionevole aspettativa. Capì che cominciava a desiderarla in modo sfrenato, anche se ciò significava tradire la fiducia di sua moglie e perdere quell' etica professionale che doveva unicamente improntare la sua relazione con L.T. Alla sera, a casa, durante la cena, a Teresa raccontò solo del facoltoso industriale corteggiatore e poi dell'assegno. Ancora una volta constatò, ma senza farsene un problema, che la moglie ascoltava in silenzio e con interesse ma che non riprendeva mai l'argomento come se una vicenda così strana fosse invece del tutto normale.

Era un lunedì mattina piovoso ma non freddo. Sebastiano si trovava nel suo ufficio per il solito ricevimento studenti quando verso le 11 vide vibrare il cellulare e materializzarsi sul display il numero di L.T. Congedò seduta stante lo studente che aveva di fronte e con un'ansia mal celata rispose. Lei era, come sempre, gentile ma la sua voce carezzevole propose, come si trattasse di un desiderio da esaudire assolutamente, un appuntamento per un giorno di quella stessa settimana, che lo scegliesse lui. L'incontro sarebbe stato a Portofino all'Hotel Pitosforo. Ancora: mentre lo informava, en passant, che aveva prenotato la suite numero 3 per l'intero week-end, lei gli chiedeva che le concedesse almeno tre ore. Sebastiano rimase interdetto. Però in pochi secondi aveva disegnato lo scenario utile per un'avventura extra moenia come se fosse stato un don Giovanni incallito. Accettò subito proponendo il venerdì a pranzo verso le 13. Perché proprio il venerdì? Perché Teresa tutti i venerdì andava a trovare una sua sorella che abitava a Siena e lui avrebbe avuto il tempo necessario per andare e venire da Portofino a Firenze senza farsi scoprire. Si stupì di come avesse accettato in quattro e quattr'otto un appuntamento così pieno di implicazioni inquietanti. Ma inquieto non era, anzi si sentiva molto ma molto allegro. Tra l'altro gli venne in mente che Richard Burton ed Elisabeth Taylor, nel loro periodo dorato, soggiornavano abitualmente al Pitosforo quando scendevano dal Kalizma, il loro yacht, a Portofino.

Il venerdì mattina arrivò in un lampo. La giornata era splendida. Un'arietta frizzante invitava ad uscire da casa e a godersi il sole. Teresa aveva preparato un'ottima prima colazione, con ogni ben di Dio, perché voleva che lui fosse a posto mentre lei sarebbe stata via da casa per tutto il giorno. Mangiarono insieme con calma e lui la trovò, insolitamente, di buon umore. Ora la guardava 
mentre beveva il caffè e mentre la luce del mattino scopriva le tante rughe che ne segnavano il viso, un viso di una donna matura, di una moglie paziente e devota, di una madre dalla generosità senza confini. Un viso ancora di una rara bellezza, una bellezza da zingara, che l'aveva fatto innamorare perdutamente quando l'aveva incontrata per caso, durante una gita in un paesino delle Dolomiti, vent'anni prima. Era vestita con il solito completino blu elettrico che indossava quando andava dalla sorella, però oggi c'era una piccola novità: portava intorno al collo il foulard rosso vermiglio che Sebastiano le aveva regalato per festeggiare la data del loro primo anno di fidanzamento. Lui invece, si era infilato dentro al completo grigio misto lana griffato e nuovo nuovo. A Teresa che gli chiedeva, senza neanche un pizzico di malizia, il motivo di tanta eleganza inusuale, lui aveva borbottato, con gli occhi bassi, una squallida bugia. Quel giorno sarebbe stato impegnato in un seduta di tesi di laurea dove avrebbe partecipato anche il rettore dell'ateneo. Uscirono di casa insieme sulla vecchia Lancia Thema, color grigio canna di fucile, che Sebastiano curava da maniaco, seguendo alcuni insegnamenti tramandatagli dal padre. Arrivarono alla stazione delle corriere in pochi minuti. Teresa, sempre sorridente mentre lo salutava con un bacio casto sulla guancia, gli augurò buona giornata accarezzandogli la testa, un gesto materno abituale tra di loro che lui subiva con gratitudine perché sapeva bene quanto affetto nascondesse. Dopo averla lasciata salire sul bus per Siena, Sebastiano imboccò il viale Guidoni per prendere da lì a poco l'autostrada per Genova.

Arrivò a Portofino verso le 12,30, senza intoppi. L'Hotel Pitosforo era un palazzotto tipicamente ligure nell'architettura e nei colori accesi. Collocato in una posizione strategica all'ingresso del porto, era circondato da un parco accuratissimo ed ornato per l'appunto da un' enorme pianta secolare di pitosforo che, in quella stagione era ancora fiorito. Lasciata l'auto nel parcheggio, Sebastiano scese lentamente a piedi verso la piazzetta antistante al mare e si trovò davanti all'hotel in leggero anticipo rispetto all'ora fissata per l'incontro con L.T. Decise così di andare nel giardino. Da là si poteva accedere alla sala da pranzo circondata da una vetrata. Si avvicinò con circospezione ed improvvisamente la vide. Era assorta, seduta ad un tavolo che le doveva consentire la vista del mare; stava fumando ed era straordinariamente bella. Si era vestita con una specie di tunica morbida che riproduceva il disegno maculato della pelliccia del leopardo, un look decisamente da cafoni se il vestito non fosse stato, invece, di un tessuto finissimo e drappeggiato in maniera morbida ed elegante da qualche stilista di grido. Questa scelta così audace era sottolineata, nella sua promettente aggressività, da un foulard color rosso vermiglio che esaltava il pallore del suo viso. Ci fu un lungo momento di sorpresa. Il rosso del foulard ebbe su Sebastiano, più o meno, lo stesso effetto devastante che la luce scesa,di colpo, dal cielo ebbe su Saulo di Tarso mentre cavalcava - pieno di cattive intenzioni- sulla via di Damasco. 
Seduta a quel tavolo ora c'era Teresa, con la sua luminosa semplicità. Sebastiano, attonito, vide scorrere al di là dei vetri, come in un film, le scene della loro vita insieme: il primo incontro in montagna, nella val Badia a Colfosco nell'estate del 1966, i loro appuntamenti da innamorati a Padova, a Bologna, a Milano, a Genova, il matrimonio celebrato in un' atmosfera francescana circondati solo dall'affetto dei familiari più stretti, gli anni faticosi vissuti in una soffitta sui tetti rossi di Firenze - in via Fiesolana 32, l'affetto, il rispetto e la leggera ironia con cui lei aveva seguito il suo lavoro di studioso placandone le ansie, la nascita delle loro figlie e poi i sorrisi di lei, stranamente sempre più silenziosi, nel corso del tempo. «Posso esserle d'aiuto, signore?». Era un giovane cameriere dell'hotel che gli rivolgeva la domanda, con garbo, per capire se doveva farlo entrare nella sala da pranzo oppure indicargli qualche altra prospettiva di azione. Sebastiano si sentì dire con naturalezza « No, grazie, ora vado via». E così fu.

Quando, dopo aver girato la chiavetta per accendere il motore, la Thema cominciò a muoversi, lenta ma sicura, lui sperimentò una specie di satori gioioso, si sentì come se fosse rinato. Era pervaso dalla voglia di correre a Firenze perché ora sapeva! Sapeva con assoluta certezza, che là avrebbe trovato la risposta a tutte le domande che il suo libro lasciava irrisolte.

$\S \S \S$

GIANFRANCO BETTIN LATES: Profesor ordinario e investigador en la Universidad de Florencia. Actualmente, escritor sobre las emociones y la vida cotidiana. 